\title{
Controlled release formulation of agrochemical pesticide based on 4-(2,4- dichlorophenoxy)butyrate nanohybrid
}

\begin{abstract}
Hybridization of beneficial organic guest with inorganic host affords scientists an opportunity to synthesize various combinations of new organic-inorganic nanohybrids with various potential applications, especially for controlled delivery of beneficial agent and storage. A new layered organic-inorganic nanohybrid material containing an agrochemical, 4-(2,4dichlorophenoxy)butyrate (DPBA) in Zn-Al-layered double hydroxide inorganic interlayer was synthesised by direct and indirect methods. Both methods yielded mesoporous-type, phase pure, well-ordered layered nanohybrids with similar basal spacing of 28.5-28.7 angstroms and organic loading of around 54.3\%. Compared to the material prepared by direct method, the ion exchanged product inherited more of the host's properties especially the pore structure and the organic moiety is also more easily released. This shows that the method of preparation plays an important role in determining the resulting physicochemical properties, in particular the release property and therefore can be used as a means to tune up the release property of the beneficial agent.
\end{abstract}

Keyword: 4-(2,4-dichlorophenoxy)butyrate; Controlled release; Herbecides; Layered double hydroxide; Nanohybrid 